\title{
A note on finite-horizon LQ problems with indefinite cost ${ }^{\star}$
}

\author{
Augusto Ferrante ${ }^{a}$, Lorenzo Ntogramatzidis ${ }^{b}$ \\ ${ }^{a}$ Dipartimento di Ingegneria dell'Informazione, Università di Padova, via Gradenigo, 6/B - 35131 Padova, Italy. \\ ${ }^{\mathrm{b}}$ Department of Mathematics and Statistics, Curtin University, Perth (WA), Australia.
}

\begin{abstract}
This paper addresses the discrete-time finite-horizon linear quadratic (LQ) optimal control problem in the case in which the quadratic forms in the performance index are not assumed to be positive semidefinite, but only symmetric. We provide a necessary and sufficient condition for the existence of an optimal control.
\end{abstract}

\section{Introduction}

This paper is concerned with the discrete-time finite-horizon LQ problem, which, loosely speaking, consists in finding a sequence $u_{t}$, with $t \in\{0,1, \ldots, T-1\}$ that minimizes a quadratic performance index

$$
J_{x_{0}, u, T}=\sum_{t=0}^{T-1}\left[\begin{array}{ll}
x_{t}^{\top} & u_{t}^{\top}
\end{array}\right]\left[\begin{array}{cc}
Q & S \\
S^{\top} & R
\end{array}\right]\left[\begin{array}{l}
x_{t} \\
u_{t}
\end{array}\right]+x_{T}^{\top} W x_{T},
$$

where $W$ and

$$
\Pi=\left[\begin{array}{cc}
Q & S \\
S^{\top} & R
\end{array}\right]
$$

are symmetric matrices, ${ }^{1}$ subject the the constraint

$$
x_{t+1}=A x_{t}+B u_{t}
$$

where the initial state $x_{0} \in \mathbb{R}^{n}$ is given.

Most of the literature deals with the so-called positive semidefinite case, in which both $\Pi$ and $W$ are assumed to be positive semidefinite matrices. Quoting even only a fraction of the relevant references in this area would be impossible;

\footnotetext{
* Corresponding author L. Ntogramatzidis. Tel. +61-8-92663143. Fax +61-8-92663197.

Email addresses: augusto@dei.unipd.it (Augusto

Ferrante), L.Ntogramatzidis@curtin.edu.au (Lorenzo Ntogramatzidis).

1 There is no loss of generality in assuming $W$ and $\Pi$ to be symmetric, as only the symmetric part of these matrices is relevant for the optimisation problem.
}

thus, we refer the readers to the monographs $[7,1,9]$. In the positive semidefinite case, no problems of existence of an optimal solution exists, since $J_{x_{0}, u, T} \geq 0$. The so-called indefinite case, i.e., where there are no sign constraints on the symmetric matrices $W$ and $\Pi$, has received far less attention in the literature. The infinite-horizon LQ problem with an indefinite quadratic form in the performance index was thoroughly investigated in [10]. In addition to presenting a characterization of the set of all symmetric solutions of the discrete-time algebraic Riccati equation, [10] also presented necessary and sufficient conditions for the existence of an optimal control. In [2], the finite LQ problem with indefinite terminal weight has been considered. Some results on the finite-horizon LQ problem were also presented in [6] for the indefinite case, as discussed in more detail below.

In this paper, we address the finite-horizon case in which all penalty matrices in the performance cost function are allowed to be indefinite. The fundamental tool used in this paper for the solution of the indefinite LQ problem is the generalized Riccati difference equation

$$
\begin{aligned}
X_{t}=Q+A^{\top} X_{t+1} A & -\left(S+A^{\top} X_{t+1} B\right) \\
\times & \times\left(R+B^{\top} X_{t+1} B\right)^{\dagger}\left(S^{\top}+B^{\top} X_{t+1} A\right),
\end{aligned}
$$

which has to be iterated backward starting from the terminal condition $X_{T}=W$. Notice that in the generalized Riccati equation (3) the Moore-Penrose pseudo inverse $(R+$ $\left.B^{\top} X_{t+1} B\right)^{\dagger}$ appears in place of the inverse. Unlike the positive semidefinite case, the finite-horizon LQ problem may not admit solutions because the performance index can be made arbitrarily negative by means of suitable control actions. Consider for example the problem of minimizing (1) subject to (2), where $A=-1, B=Q=S=W=0, R=-1$ 
and $T=0$. In this case, the cost function to be minimized, regardless of the initial condition, is $J_{x_{0}, u}=-u_{0}^{2}$, which can obviously be made arbitrarily negative by choosing $u_{0}$ suitably.

As already anticipated, some results on indefinite finitehorizon LQ problems have also been presented in [6]. In particular, Theorem 9.2.1 in [6] gives a condition for the solvability of such problem by considering the standard Riccati recursion that differs from (3) only as the matrix inverse term $\left(R+B^{\top} X_{t+1} B\right)^{-1}$ appears in place of the MoorePenrose pseudo inverse term $\left(R+B^{\top} X_{t+1} B\right)^{\dagger}$. In the positive semidefinite case, it appears to be rather natural to consider the matrix inverse in the Riccati recursion (3). Indeed, by just adopting some additional mild assumptions (for example, by assuming $R>0$ instead of simply $R \geq 0$ ) it is easy to guarantee the invertibility of the matrix $R+B^{\top} X_{t} B$ at each step of the iteration. Things are different in the indefinite case, because there appear to be no natural assumptions on the problem data that guarantee the invertibility of the matrix $R+B^{\top} X_{t} B$ at each step of the iteration. This means that the assumption $\operatorname{det}\left(R+B^{\top} X_{t} B\right) \neq 0$ for all $t \in\{0, \ldots, T\}$ can only be tested by iterating the Riccati equation, and it is therefore an assumption on the solution of the problem and not on the problem data. For this reason, the generalized Riccati equation (3) seems to represent a more natural tool in the context of indefinite LQ problems than the standard Riccati difference equation.

Another (rather counterintuitive) aspect that is peculiar to the indefinite case is the fact that the optimal control may fail to exist (i.e., for a certain $x_{0}$, the index (1) can be made arbitrarily negative) for a problem with data $A, B, \Pi, W$ and time horizon $T$, while for a problem with the same data $A, B, \Pi, W$ and time horizon $T_{1}>T$, an optimal control does exist. Consider e.g. the problem of minimizing (1) subject to (2), where $A=\left[\begin{array}{ll}0 & 0 \\ 0 & 0\end{array}\right], B=\left[\begin{array}{l}1 \\ 0\end{array}\right], Q=\left[\begin{array}{cc}10 & 0 \\ 0 & 10\end{array}\right], S=\left[\begin{array}{l}0 \\ 1\end{array}\right]$, $W=\left[\begin{array}{ll}0 & 0 \\ 0 & 0\end{array}\right]$ and $R=0$. In this case, partitioning $x_{0}$ as $x_{0}=$ $\left[\begin{array}{l}x_{0,1} \\ x_{0,2}\end{array}\right]$, if $T=1$ we find

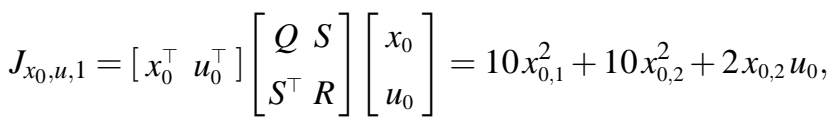

which, for any $x_{0}$ such that $x_{0,2} \neq 0$, can be made arbitrarily small by suitably choosing $u_{0} \in \mathbb{R}$. On the other hand, if $T=2$, we find

$$
J_{x_{0}, u, 2}=10 x_{0,1}^{2}+9\left(x_{0,2}^{2}+u_{0}^{2}\right)+\left(x_{0,2}+u_{0}\right)^{2} \geq 0,
$$

whose minimum cannot be made arbitrarily negative.

This consideration completes the picture drawn in [6, Theorem 9.2.1], and highlights the fact that the indefinite case is much richer than the semidefinite case.
From this discussion, we can also conclude that a fundamental problem in the context of indefinite finite-horizon LQ problems, which is the problem considered in this paper, is the following.

Problem 1.1 Let $T \in \mathbb{N}$. Let $A \in \mathbb{R}^{n \times n}$ and $B \in \mathbb{R}^{n \times m}$, and consider the linear state difference equation (2) with $x_{0} \in$ $\mathbb{R}^{n}$. Find necessary and sufficient conditions under which, for any time horizon $\{0, \ldots, N\} \subseteq\{0, \ldots, T\}$, there exists a sequence $u_{t}$, with $t \in\{0,1, \ldots, N-1\}$ which minimizes the performance index $J_{x_{0}, u, N}$ in (1). Under such conditions, find all the control trajectories optimizing the index (1).

\section{Main result}

In the positive semidefinite case, i.e., when $\Pi=\Pi^{\top} \geq 0$ and $W=W^{\top} \geq 0$, Problem 1.1 always admits solutions, and such solutions are given by

$$
u_{t}=-K_{t} x_{t}+G_{t} v_{t}
$$

where $X_{t}$ is the solution of the generalized Riccati recursion (3) with the terminal condition $X_{T}=W, K_{t} \stackrel{\text { def }}{=}(R+$ $\left.B^{\top} X_{t+1} B\right)^{\dagger}\left(S^{\top}+B^{\top} X_{t+1} A\right), G_{t} \stackrel{\text { def }}{=}\left[I_{m}-\left(R+B^{\top} X_{t+1} B\right)^{\dagger}(R+\right.$ $\left.B^{\top} X_{t+1} B\right)$ ] and $v_{t}$ is arbitrary, see e.g. [11]. Matrix $G_{t}$ is the orthogonal projector onto the linear space of vectors that can be added to the optimal control $u_{t}$ without affecting optimality. It is easily seen that, when $W$ and $\Pi$ are both positive semidefinite, for all $t \in\{0,1, \ldots, T\}$ the following facts automatically hold:

- $X_{t}$ is positive semidefinite;

- $R+B^{\top} X_{t} B$ is positive semidefinite;

- $\operatorname{ker}\left(R+B^{\top} X_{t} B\right) \subseteq \operatorname{ker}\left(S+A^{\top} X_{t} B\right)$,

see also Remark 2.1 below. In the indefinite case, none of these facts hold in general. Consider for example Problem 1.1 with $A=\left[\begin{array}{cc}0 & -3 \\ 0 & 0\end{array}\right], B=Q=\left[\begin{array}{ll}0 & 0 \\ 0 & 0\end{array}\right], S=\left[\begin{array}{ll}1 & 0 \\ 0 & 1\end{array}\right], R=\left[\begin{array}{cc}-4 & 0 \\ 0 & 0\end{array}\right]$ and $W=\left[\begin{array}{cc}-1 & -1 \\ -1 & 0\end{array}\right]$. At $t=T$, we have $X_{T}=W$, which is indefinite; moreover, $R+B^{\top} X_{T} B=\operatorname{diag}\{-4,0\}$ is negative semidefinite and $\operatorname{span}\left\{\left[\begin{array}{l}0 \\ 1\end{array}\right]\right\}=\operatorname{ker}\left(R+B^{\top} X_{T} B\right) \nsubseteq$ $\operatorname{ker}\left(S+A^{\top} X_{T} B\right)=\{0\}$

In order to establish the main result of this paper, we need the following simple technical result.

Lemma 2.1 Let $R$ be symmetric. Matrix $M=\left[\begin{array}{ccc}S R^{\dagger} S^{\top} & S \\ S^{\top} & R\end{array}\right]$ is positive semidefinite if and only if $R \geq 0$ and $\operatorname{ker} R \subseteq \operatorname{ker} S$.

Proof: If $R \geq 0$ and $\operatorname{ker} R \subseteq \operatorname{ker} S$, we can write

$$
M=\left[\begin{array}{l}
S \\
R
\end{array}\right] R^{\dagger}\left[\begin{array}{ll}
S^{\top} & R
\end{array}\right]
$$


where we have used the fact that $\operatorname{ker} R \subseteq \operatorname{ker} S$. Since $R \geq 0$, such is also $R^{\dagger}$, which implies $M \geq 0$.

If $M \geq 0$, it admits a factorization $M=\left[\begin{array}{cc}S R^{\dagger} S^{\top} & S \\ S^{\top} & R\end{array}\right]=$ $\left[\begin{array}{l}C^{\top} \\ D^{\top}\end{array}\right]\left[\begin{array}{ll}C & D\end{array}\right]$ in terms of two matrices of suitable sizes $C$ and $D$, so that $R=D^{\top} D$ is positive semidefinite. To prove that $\operatorname{ker} R \subseteq \operatorname{ker} S$, take $\omega \in \operatorname{ker} R$. This implies that $\omega \in \operatorname{ker}\left(D^{\top} D\right)=\operatorname{ker} D$, so that also $S \omega=C^{\top} D \omega=0$.

We are now ready to present the main result of this paper.

Theorem 2.1 Let $\left\{X_{t}\right\}_{t \in\{0,1, \ldots, T\}}$ be the sequence of matrices arising from (3) with $X_{T}=W$. Problem 1.1 admits solutions for any initial state $x_{0} \in \mathbb{R}^{n}$ if and only if

$$
R+B^{\top} X_{t} B \geq 0
$$

and

$$
\operatorname{ker}\left(R+B^{\top} X_{t} B\right) \subseteq \operatorname{ker}\left(S+A^{\top} X_{t} B\right)
$$

for all $t \in\{0,1, \ldots, T\}$. In the case in which Problem 1.1 admits solutions, the optimal control is parameterized in terms of the arbitrary vector $v_{t}$ by

$$
u_{t}=-K_{t} x_{t}+G_{t} v_{t},
$$

where $K_{t}=\left(R+B^{\top} X_{t+1} B\right)^{\dagger}\left(S^{\top}+B^{\top} X_{t+1} A\right)$ and $G_{t}=\left[I_{m}-\right.$ $\left.\left(R+B^{\top} X_{t+1} B\right)^{\dagger}\left(R+B^{\top} X_{t+1} B\right)\right]$, and the optimal cost is

$$
J^{*}=x_{0}^{\top} X_{0} x_{0} .
$$

Proof: We begin by proving sufficiency. Let $\left\{X_{t}\right\}_{t \in\{0,1, \ldots, T\}}$ be the sequence of matrices obtained iterating (3) with $X_{T}=$ $W$, and such that (5) and (6) hold for all $t \in\{0,1, \ldots, T\}$. Since

$$
\sum_{t=0}^{T-1}\left(x_{t+1}^{\top} X_{t+1} x_{t+1}-x_{t}^{\top} X_{t} x_{t}\right)+x_{0}^{\top} X_{0} x_{0}-x_{T}^{\top} X_{T} x_{T}=0,
$$

adding (9) to (1) and using (2) yields

$$
\begin{aligned}
J_{x_{0}, u, T} & =\sum_{t=0}^{T-1}\left[x_{t}^{\top} u_{t}^{\top}\right]\left[\begin{array}{ll}
Q+A^{\top} X_{t+1} A-X_{t} & S+A^{\top} X_{t+1} B \\
S^{\top}+B^{\top} X_{t+1} A & R+B^{\top} X_{t+1} B
\end{array}\right]\left[\begin{array}{l}
x_{t} \\
u_{t}
\end{array}\right] \\
& +x_{T}^{\top}\left(W-X_{T}\right) x_{T}+x_{0}^{\top} X_{0} x_{0},
\end{aligned}
$$

and, in view of (3),

$M_{t+1} \stackrel{\text { def }}{=}\left[\begin{array}{cc}Q+A^{\top} X_{t+1} A-X_{t} & S+A^{\top} X_{t+1} B \\ S^{\top}+B^{\top} X_{t+1} A & R+B^{\top} X_{t+1} B\end{array}\right]=\left[\begin{array}{cc}S_{t} R_{t}^{\dagger} S_{t}^{\top} & S_{t} \\ S_{t}^{\top} & R_{t}\end{array}\right]$

is positive semidefinite, where $S_{t} \stackrel{\text { def }}{=} S+A^{\top} X_{t+1} B$ and $R_{t} \stackrel{\text { def }}{=}$ $R+B^{\top} X_{t+1} B$. Since $\operatorname{ker} R_{t} \subseteq \operatorname{ker} S_{t}$ in view of (6), $M_{t+1}$ admits a factorization of the form

$$
M_{t+1}=\left[\begin{array}{c}
S_{t} \\
R_{t}
\end{array}\right] R_{t}^{\dagger}\left[\begin{array}{ll}
S_{t}^{\top} & R_{t}
\end{array}\right]
$$

and $R_{t}$ is positive semidefinite for all $t \in\{0,1, \ldots, T\}$. Hence, (10) takes the form

$J_{x_{0}, u, T}=\sum_{t=0}^{T-1}\left\|\left[R_{t}^{1 / 2}\right]^{\dagger} S_{t}^{\top} x_{t}+R_{t}^{1 / 2} u_{t}\right\|_{2}^{2}+x_{0}^{\top} X_{0} x_{0}$.

Since the control only appears in the sum, which is a nonnegative quantity, and does not affect the term $x_{0}^{\top} X_{0} x_{0}$, Problem 1.1 admits a solution because the sum can be annihilated choosing

$$
\left[R_{t}^{1 / 2}\right]^{\dagger} S_{t}^{\top} x_{t}+R_{t}^{1 / 2} u_{t}=0,
$$

whose solutions are parameterized in $v_{t}$ by the feedback control (7). The optimal state trajectory is now given by the closed-loop dynamics

$$
x_{t+1}=\left(A-B K_{t}\right) x_{t}+B G_{t} v_{t} .
$$

Now we prove necessity of conditions (5) and (6). We show that when either of these two conditions does not hold, it is always possible to find $N \leq T$ and $x_{0} \in \mathbb{R}^{n}$ such that $J_{x_{0}, u, N}$ can be made arbitrarily negative by suitably selecting the input sequence. Let $s$ represent the largest value of $t \in$ $\{0, \ldots, T\}$ in which either $R+B^{\top} X_{t} B$ becomes indefinite or (6) ceases to hold. In view of Lemma 2.1, this means that $M_{s}$ in (11) for $t+1=s$ is indefinite. Let $T_{1} \stackrel{\text { def }}{=} T-s+1$, and consider the index $J_{x_{0}, u, T_{1}}$, which may be written as

$J_{x_{0}, u, T_{1}}=x_{0}^{\top} X_{s-1} x_{0}+\left[\begin{array}{ll}x_{0}^{\top} & u_{0}^{\top}\end{array}\right] M_{s}\left[\begin{array}{c}x_{0} \\ u_{0}\end{array}\right]+\sum_{t=1}^{T-s}\left[\begin{array}{ll}x_{t}^{\top} & u_{t}^{\top}\end{array}\right] M_{t+s}\left[\begin{array}{l}x_{t} \\ u_{t}\end{array}\right]$.

Since $M_{t+s}$ is positive semidefinite, it can be written in the form (11), and by using the same control as in (12), we can make $\sum_{t=1}^{T-s}\left[x_{t}^{\top} u_{t}^{\top}\right] M_{t+s}\left[\begin{array}{l}x_{t} \\ u_{t}\end{array}\right]$ equal to zero (for any $u_{0}$ ). On the other hand, $x_{0}^{\top} X_{s-1} x_{0}$ is bounded from below by $\lambda_{\text {min }}\left(X_{s-1}\right)\left\|x_{0}\right\|^{2}$, where $\lambda_{\text {min }}\left(X_{s-1}\right)$ denotes the smallest eigenvalue of $X_{s-1}$. Hence, it is sufficient to show that for at least one initial condition $x_{0} \in \mathbb{R}^{n}$, the quantity $\left[\begin{array}{ll}x_{0}^{\top} & u_{0}^{\top}\end{array}\right] M_{s}\left[\begin{array}{l}x_{0} \\ u_{0}\end{array}\right]$ can be made arbitrarily negative by suitably selecting $u_{0} \in \mathbb{R}^{m}$. We have

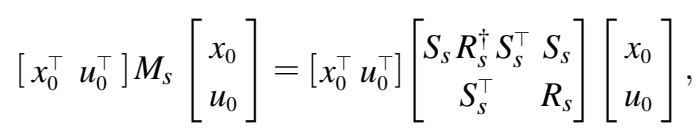

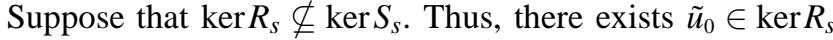
but $\tilde{u}_{0} \notin \operatorname{ker} S_{s}$. Let $x_{0}=S_{s} \tilde{u}_{0}$. Finally, let $u_{0}=\alpha \tilde{u}_{0}$. With 
these definitions, we find

$$
\begin{aligned}
{\left[\begin{array}{ll}
x_{0}^{\top} & u_{0}^{\top}
\end{array}\right] M_{s}\left[\begin{array}{c}
x_{0} \\
u_{0}
\end{array}\right] } & =x_{0}^{\top} S_{s} R_{s}^{\dagger} S_{s}^{\top} x_{0}+u_{0}^{\top} R_{s} u_{0}+2 x_{0}^{\top} S_{s}^{\top} u_{0} \\
& =x_{0}^{\top} S_{s} R_{s}^{\dagger} S_{s}^{\top} x_{0}+2 \alpha \tilde{u}_{0}^{\top} S_{s}^{\top} S_{s} \tilde{u}_{0},
\end{aligned}
$$

which can be made arbitrarily small for the chosen initial condition $x_{0}$, by choosing the parameter $\alpha$ accordingly, since $\tilde{u}_{0}^{\top} S_{s}^{\top} S_{s} \tilde{u}_{0} \neq 0$.

Now, suppose that $R_{S}$ is not positive semidefinite. Then, it is clear that, for any given initial condition $x_{0} \in \mathbb{R}^{n}$,

$$
\left[\begin{array}{ll}
x_{0}^{\top} & u_{0}^{\top}
\end{array}\right] M_{s}\left[\begin{array}{l}
x_{0} \\
u_{0}
\end{array}\right]=x_{0}^{\top} S_{s} R_{s}^{\dagger} S_{s}^{\top} x_{0}+u_{0}^{\top} R_{s} u_{0}+2 x_{0}^{\top} S_{s}^{\top} u_{0}
$$

can be made arbitrarily negative by suitably selecting $u_{0}$. We can conclude that there exists a horizon $\left\{0, \ldots, T_{1}\right\}$ with $T_{1} \leq T$ for which the optimal control does not exist.

Remark 2.1 Now we are in a position to better understand the observation that we made earlier concerning the difference with the positive semidefinite case. When $W$ and $\Pi$ are positive semidefinite, it is easy to see by induction that $\left\{X_{t}\right\}_{t \in\{0, \ldots, T\}}$ is a sequence of positive semidefinite matrices. Indeed, $X_{T} \geq 0$. Moreover,

$$
\left[\begin{array}{cc}
Q+A^{\top} X_{t+1} A-X_{t} & S_{t} \\
S_{t}^{\top} & R_{t}
\end{array}\right]=\Pi+\left[\begin{array}{c}
A^{\top} \\
B^{\top}
\end{array}\right] X_{t+1}\left[\begin{array}{ll}
A & B
\end{array}\right] \geq 0 .
$$

Since the right hand-side of (3) is exactly the Schur generalized complement of the matrix in the left hand-side of the latter, we find that $X_{t} \geq 0$ as well. Thus, $\left\{X_{t}\right\}_{t \in\{0, \ldots, T\}}$ is a sequence of positive semidefinite matrices, and such is therefore also $\left\{R+B^{\top} X_{t} B\right\}_{t \in\{0, \ldots, T\}}$. The positive semidefiniteness of the matrix in (14) implies that

$$
\operatorname{ker}\left(R+B^{\top} X_{t} B\right) \subseteq \operatorname{ker}\left(S+A^{\top} X_{t} B\right),
$$

for all $t \in\{0, \ldots, T\}$, which is now a consequence of (14) and automatically holds.

Consider now the so-called generalized discrete algebraic Riccati equation $\operatorname{GDARE}(\Sigma)$, defined as

$X=A^{\mathrm{T}} X A-\left(A^{\mathrm{T}} X B+S\right)\left(R+B^{\mathrm{T}} X B\right)^{\dagger}\left(B^{\mathrm{T}} X A+S^{\mathrm{T}}\right)+Q$.

This algebraic equation, subject to the constraint

$$
\operatorname{ker}\left(R+B^{\mathrm{T}} X B\right) \subseteq \operatorname{ker}\left(A^{\mathrm{T}} X B+S\right)
$$

is usually referred to as constrained generalized discrete algebraic Riccati equation CGDARE $(\Sigma)$. The properties of this equation in relation to optimal LQ problems have been discussed in the positive semidefinite cases in $[3-5,8]$. Some important properties of $\operatorname{CGDARE}(\Sigma)$ - but not within the framework of optimal control problems - were studied in [12] also in the indefinite case.
We observe the following important facts:

- If $W=X$, where $X$ is a solution of $\operatorname{CGDARE}(\Sigma)$ such that $R+B^{\mathrm{T}} X B$ is positive semidefinite, from the iteration (3) we have that $X_{t}=X$ for any $t \in\{0, \ldots, T\}$ and any $T>0$. Thus, in this case an optimal control exists in any horizon, and is stationary. In other words, for any $T>0$, the optimal control is $u_{t}=-K_{X} x_{t}+G_{X} v_{t}$, where $K_{X}=\left(R+B^{\top} X B\right)^{\dagger}\left(S^{\top}+B^{\top} X A\right)$ and $G_{X}=\left[I_{m}-\right.$ $\left.\left(R+B^{\top} X B\right)^{\dagger}\left(R+B^{\top} X B\right)\right]$. The optimal cost equals $J^{*}=$ $x_{0}^{\top} X x_{0}$, and does not depend on the length of the time horizon;

- if $W \geq X$, where $X$ is a solution of $\operatorname{CGDARE}(\Sigma)$ such that $R+B^{\mathrm{T}} X B$ is positive semidefinite, an optimal control exists in any horizon. Indeed, in such case the cost is bounded from below by the cost that corresponds to the case in which $W$ is itself a solution of CGDARE $(\Sigma)$ such that $R+B^{\mathrm{T}} W B \geq 0$, which as we already noticed cannot be made arbitrarily negative; as a particular case, if $W=0$ and $\operatorname{CGDARE}(\Sigma)$ admits a negative semidefinite solution $X$ such that $R+B^{\mathrm{T}} X B$ is positive semidefinite, Problem 1.1 admits an optimal control in any horizon.

\section{References}

[1] B.D.O. Anderson and J.B. Moore. Optimal Control: Linear Quadratic Methods. Prentice Hall International, London, 1989.

[2] G. Bilardi, and A. Ferrante, "The Role of Terminal Cost/Reward in Finite-Horizon Discrete-Time LQ Optimal Control", Linear Algebra and its Applications, 425:323-344, 2007.

[3] A. Ferrante, and L. Ntogramatzidis, "The generalized discrete algebraic Riccati equation in linear-quadratic optimal control". Automatica, 49(2):471-478, 2013.

[4] A. Ferrante, and L. Ntogramatzidis, "The extended symplectic pencil and the finite-horizon LQ problem with two-sided boundary conditions", IEEE Transactions on Automatic Control, 58(8), 21022107, 2013.

[5] A. Ferrante, and L. Ntogramatzidis, "A reduction technique for discrete generalized algebraic and difference Riccati equations", Linear and Multilinear Algebra, 62(11):1460-1474, 2014.

[6] B. Hassibi, A.H. Sayed, and T. Kailath. Indefinite-Quadratic Estimation and Control, A Unified Approach to $\mathrm{H}^{2}$ and $\mathrm{H}^{\infty}$ Theories. SIAM, Philadelphia, 1999.

[7] H. Kwakernaak and R. Sivan. Linear Optimal Control Systems John Wiley \& Sons, New York, 1972.

[8] V. Ionescu, C. Oară, and M. Weiss. Generalized Riccati theory and robust control, a Popov function approach. Wiley, 1999.

[9] F.L. Lewis and V. Syrmos. Optimal Control. John Wiley \& Sons, New York, 1995.

[10] A.C.M. Ran and H.L. Trentelman. Linear quadratic problems with indefinite cost for discrete time systems. SIAM Journal on Matrix Analysis and Applications, 14(3):776-797, July 1993.

[11] D. Rappaport and L.M. Silverman. Structure and stability of discrete-time optimal systems. IEEE Transactions on Automatic Control, 16:227-233, 1971.

[12] A.A. Stoorvogel and A. Saberi. The discrete-time algebraic Riccati equation and linear matrix inequality. Linear Algebra and its Applications, 274:317-365, 1998. 